\title{
Cocaine-Induced Bronchospasm Mimicking Acute Asthma Exacerbation
}

\author{
Christine Y Zhou, BS; Melissa Ricker, PA-C; and Vikas Pathak, MD
}

\begin{abstract}
Cocaine can cause a myriad of changes in the lung, which can range from bronchoconstriction to destruction of the alveolar-capillary membrane and acute lung injury. Cocaine-induced bronchospasm is a diagnosis of exclusion that should be considered when the clinical presentation of acute hypoxic and hypercapneic respiratory failure cannot be explained by chronic obstructive pulmonary disease or asthma exacerbation, anaphylaxis to food or medications, exercise, or infection. Here, we present two patients with acute hypoxic and hypercapneic respiratory failure that was ultimately attributed to cocaine use shortly prior to symptom onset.
\end{abstract}

Keywords: Cocaine; Bronchospasm, Asthma exacerbation

$\mathrm{C}$ ocaine is an alkaloid with unique chemical properties. Cocaine abuse is seen all over the world, and it can cause toxicity in multiple organs due to its sympathomimetic and stimulant effect on the central nervous system. Cocaine can be inhaled, smoked, or injected intravenously. The toxicity can occur in cardiac (coronary vasospasm, myocardial infarction), central nervous system (stroke), and respiratory system (alveolitis, hemorrhage). Cocaine can cause several pulmonary complications like eosinophilic pneumonia, crack lung, pneumothorax, pneumomediastinum, foreign body granuloma, pulmonary hypertension, or organizing pneumonia. One such pulmonary complication that is underappreciated is bronchospasm. We present two patients with history of substance abuse who presented to the emergency department (ED) with acute hypoxic and hypercapneic respiratory failure and were diagnosed with cocaine-induced bronchospasm.

\section{Case One}

A woman, aged 51 years, was on her porch when she became acutely short of breath. Emergency medical services found her tachypneic on room air with an oxygen saturation in the 70 s. She was placed on continuous non-invasive positive pressure ventilation machine, given an albuterol nebulizer and intravenous methyl prednisone, and transferred to the ED. The patient had a past medical history of asthma, essential hypertension, polysubstance abuse, and medical non-compliance.

On presentation, the patient was in acute respiratory distress using her accessory muscles of respiration; her vitals revealed tachycardia (heart rate $>110 / \mathrm{min}$ ), tachypnea (respiratory rate of $35 / \mathrm{min}$ on non-invasive mechanical ventilation), and hypoxia $\left(\mathrm{O}_{2}\right.$ saturation $85 \%$ on $100 \%$ fraction of inspired oxygen $\left.\left[\mathrm{FIO}_{2}\right]\right)$. Chest examination revealed bilateral diffuse expiratory wheeze. There was no evidence of track marks, clubbing, or nasal erythema or perforation. Her arterial blood gas $(\mathrm{ABG})$ prior was $\mathrm{pH}$ 7.2, partial pressure of carbon dioxide $\left(\mathrm{pCO}_{2}\right) 84 \mathrm{mmHg}$, partial pressure of oxygen $\left(\mathrm{pO}_{2}\right)$ $52 \mathrm{mmHg}$, and hydrogencarbonate $\left(\mathrm{HCO}_{3}\right) 21 \mathrm{mmol} / \mathrm{L}$. She was therefore intubated for acute hypoxic and hypercapneic respiratory failure. Post-intubation and sedation, her ABG was as follows: $\mathrm{pH} 7.34, \mathrm{pCO}_{2} 36.9 \mathrm{mmHg}, \mathrm{HCO}_{3} 19.9$ $\mathrm{mmol} / \mathrm{L}$, and $\mathrm{O}_{2}$ saturation $99 \%$. Laboratory testing revealed an elevated troponin and creatinine, all others were unremarkable. Urine drug screen (UDS) revealed cocaine.
Corresponding Author: Vikas Pathak, MD, FACP, FCCP,ATSF, WakeMed Health and Hospitals, Pulmonary/Critical Care, 3000 New Bern Avenue, Raleigh, NC 27519, Tel: (57I) 230-4087, Email: drvikaspathak@gmail.com
Received: June 18,2018

Revised: November 14, 2018

Accepted: November 20, 2018

doi: $10.3121 / \mathrm{cmr} .2019 .1447$ 
Electrocardiogram (EKG) demonstrated old and stable left bundle branch block when compared to a prior EKG. Chest radiography (CXR) and transthoracic echocardiogram (TTE) showed no acute pathology.

She was treated empirically for asthma exacerbation with steroids, bronchodilators, and empiric antibiotics. Over the next 24 hours, her wheezing resolved, and she was successfully extubated. Her creatinine normalized. After extubation, she confirmed snorting cocaine prior to the beginning of the symptoms. She denied having a formal diagnosis of asthma, pulmonary function testing, or being on any maintenance therapy in the past. A next day stress test by the Cardiology service failed to demonstrate any ischemia. The elevated troponin was attributed to cocaine-induced coronary artery vasospasm. In the setting of unclear and quick onset, negative CXR findings, and rapid improvement, etiology of her respiratory failure was diagnosis as cocaine-induced bronchoconstriction.

\section{Case Two}

A woman, aged 59 years, was traveling in the car with friends when she became acutely short of breath, prompting the friends to drop her off at the ED. She had a past medical history of obesity, chronic obstructive pulmonary disease (COPD; although no pulmonary function test available), asthma, obstructive sleep apnea not compliant with nightly continuous positive airway pressure (CPAP), multiple episodes of respiratory failure requiring intubation, and polysubstance abuse. Upon initial evaluation in the ED, she was tachypneic with accessory muscle use. She denied recent fever, productive cough, or hemoptysis. She was not using her supplemental oxygen in the car and denied using her CPAP the night prior. She acknowledged nasal insufflations of cocaine prior to getting into her friend's car. She was given albuterol nebulizer treatment and intravenous methyl prednisone with no improvement.

Due to acute respiratory distress, the patient was intubated in the ED. Post-intubation ABG (an hour after intubation) revealed $\mathrm{pH} 7.36, \mathrm{pCO}_{2} 45.7 \mathrm{mmHg}, \mathrm{pO}_{2} 354 \mathrm{mmHg}, \mathrm{HCO}_{3}$ $26.3 \mathrm{mmol} / \mathrm{L}$, and $\mathrm{O}_{2}$ saturation of $100 \%$. Laboratory testing demonstrated an elevated troponin, all others unremarkable. UDS revealed cocaine. And EKG was unremarkable, and CXR was normal. Computerized tomography of the chest was obtained, demonstrating bilateral lower lobe atelectasis. Echocardiogram showed ejection fraction of $55 \%$ to $60 \%$ with a calcification on the left coronary cusp of the aortic valve with no evidence of stenosis or regurgitation. The patient was again treated empirically for acute asthma exacerbation with steroids, bronchodilators, and antibiotics. She was extubated within 48 hours.

Given the doubtful diagnosis of COPD in the absence of pulmonary function test, no increase in volume or change in color of sputum production or fever, quick onset, and no wheezing on examination, COPD and asthma exacerbations seemed unlikely. Her presentation was attributed to her admitted cocaine use.

\section{Discussion}

While cocaine use has decreased over the past decade, the National Survey on Drug Use and Health still estimates about 1.5 million current users aged 12 years or older with 913,000 meeting the criteria for abuse or dependence. ${ }^{1}$ Cocaine is the most common cause of drug-related ED visits with nearly 500,000 visits annually for complications related to abuse. ${ }^{2,3}$ While chest pain is the most frequent cocaine-related complaint in the ED, pulmonary symptoms are also significant. ${ }^{4}$ Complications include pulmonary edema, interstitial pneumonitis, pulmonary hypertension, diffuse alveolar hemorrhage, barotrauma, thermal airway injury, hilar lymphadenopathies, crack lung and eosinophilic lung disease, infection, and aspiration pneumonia. ${ }^{5}$

The pulmonary complications of cocaine abuse are influenced by the route, amount, purity of cocaine, and presence of other toxins. Oral or nasal insufflation is the most direct method that exposes the lung parenchyma to harmful chemicals as well as thermal damage. ${ }^{6,7}$ The extent of injury to the lung parenchyma may differ based on the technique of insufflation, breath holding, and the amount of cocaine. ${ }^{4,6} \mathrm{With}$ the average size of particles being $2.3 \mu \mathrm{m}$, cocaine can easily deposit within pulmonary alveoli, leading to significant accumulation of carbon pigment and macrophages. ${ }^{3}$ Data on the effects of this particle accumulation on the diffusing capacity of the alveolar membranes or other pulmonary mechanics is still somewhat inconclusive with contradicting results. ${ }^{6-8}$

One of the more severe complications secondary to direct inhalation is known as crack lung, where inhalation of freebase cocaine results in the accumulation of eosinophils, cytokines, and other participants of the inflammatory cascade. ${ }^{3,9}$ Biopsy results would show diffuse alveolar damage with alveolar hemorrhage, and tissue findings would showcase elevated macrophages and eosinophils. ${ }^{3,6}$ Radiographic findings include diffuse perihilar and interstitial opacities that may have a ground-glass appearance. A bronchoalveolar lavage can confirm the diagnosis if radiographic findings are not conclusive. ${ }^{3}$ Treatment with empiric steroids or other immunosuppressive agents remains controversial. ${ }^{10}$

Insufflated cocaine can also cause mucosal lesions of the nose and upper airways. ${ }^{11}$ If cocaine abuse is suspected, a close facial, nasal, and oral exam should be performed. These lesions may be due to direct injury to the mucosa from cocaine ingestion, worsened by the potent vasoconstrictive effects that cocaine exerts as inhibition of catecholamine reuptakes leads to excessive stimulation of central sympathetic outflow. ${ }^{4}$

Typically, cocaine is thought to affect the lungs via a similar sympathetic outflow effect. As catecholamine levels rise from cocaine's effects, the stimulation of alpha-receptors within 
bronchial smooth muscles results in smooth muscle relaxation with subsequent widening of the airways. ${ }^{4}$ However, in patients with a history of obstructive lung disease, such as both of our described patients, cocaine can commonly cause an acute exacerbation of respiratory symptoms. ${ }^{7,12,13}$ Bronchoconstriction results from the abnormal contraction of the smooth muscle within the bronchi directly triggered by cocaine within the airways, resulting in an acute airway narrowing. ${ }^{4,6}$ This effect is not seen in patients who inject cocaine intravenously. ${ }^{7}$ The degree of obstruction is variable and may progress from simple wheezing to respiratory failure, as it did for our patients. Imaging characteristics are similar to patients with asthma exacerbations. ${ }^{3}$

Obstruction leads to retention of $\mathrm{CO}_{2}$, explaining the resulting hypercarbia that was seen in our patients. These exacerbations may also be worsened by the lack of medical compliance found in this patient population; their underlying disease process is likely uncontrolled at baseline. ${ }^{13}$

Overall management is similar to asthma or COPD exacerbation. Screening is typically performed with a UDS. However, it is worth noting that the bronchoconstrictive effects of cocaine inhalation may occur almost immediately, and thus a urine sample may be falsely negative at time of presentation to the ED. Blood testing is typically also available, but unnecessary. ${ }^{4}$ Administration of a beta- 2 adrenergic agonist and corticosteroid can treat bronchoconstriction and airway edema. On physical examination, wheezing or evidence of hemorrhage from the respiratory tract may be evident. Early recognition of respiratory failure should be treated with timely intubation and ventilator support and has a significant effect on the length of hospitalization. ${ }^{13}$

\section{Conclusion}

Providers must include cocaine-induced bronchospasm in their differential of acute hypoxic and hypercapneic respiratory failure. UDS is a relatively inexpensive test and should be obtained on any patient who has developed acute respiratory failure when the diagnosis seems unclear, or when drug abuse is suspected. The presence of an underlying lung disease such as asthma or COPD can increase the risk of acute bronchoconstriction and worsen the severity of a patient's symptoms. Promptly recognizing and treating acute respiratory failure is critical for the survival of patients seen and treated in the acute care setting.

\section{References}

1. Center for Behavioral Health Statistics and Quality. Behavioral health trends in the United States: Results from the 2014 National Survey on Drug Use and Health. HHS Pulication No. SMA 15-4927, NSDUH Ser. H-50. 64. 2015. Available at: http://www.samhsa.gov/data/. Accessed June 11, 2018.

2. National Institute on Drug Abuse. Cocaine. 2016. Available at: https:/www.drugabuse.gov/publications/research-reports/ cocaine/what-cocaine. Accessed June 11, 2018.
3. Restrepo CS, Carrillo JA, Martinez S, Ojeda P, Rivera AL, Hatta A. Pulmonary complications from cocaine and cocaine-based substances: imaging manifestations. Radiographics. 2007;27(4):941-956.

4. Goldstein RA, DesLauriers C, Burda AM. Cocaine: history, social implications, and toxicity-a review. Dis Mon. 2009;55(1):638.

5. Tashkin DP, Kleerup EC, Koyal SN, Marques JA, Goldman, MD. Acute effects of inhaled and IV cocaine on airway dynamics. Chest 1996;110(4):904-910.

6. Walkenstein MD, Haim DY, Lippmann ML, Goldberg SK. The pulmonary complications of crack cocaine. A comprehensive review. Chest. 1995;107(1);233-240.

7. Glauser J, Queen JR. An overview of non-cardiac cocaine toxicity. J Emerg Med. 2007;32(2):181-186.

8. Kleerup EC, Koyal SN, Marques-Magallanes JA, Goldman MD, Tashkin DP. Chronic and acute effects of 'crack' cocaine on diffusing capacity, membrane diffusion, and pulmonary capillary blood volume in the lung. Chest. 2002;122(2):629638.

9. Baldwin GC, Buckley DM, Roth MD, Kleerup EC, Tashkin DP. Acute activation of circulating polymorphonuclear neutrophils following in vivo administration of cocaine: A potential etiology for pulmonary injury. Chest. 1997;111(3):698-705.

10. Kissner DG, Lawrence WD, Selis JE, Flint A. Crack lung: pulmonary disease caused by cocaine abuse. Am Rev Respir Dis. 1987;136(5):1250-1252.

11. Woyceichoski IE, de Arruda EP, Resende LG, Machado MA, Gregio AM, Azevedo LR, De Lima AA. Cytomorphometric analysis of crack cocaine effects on the oral mucosa. Oral Surg Oral Med Oral Pathol Oral Radiol Endod. 2008;105(6):745-749.

12. Rome LA, Lippmann ML, Dalsey WC, Taggart P, Pomerantz S. Prevalence of cocaine use and its impact on asthma exacerbation in an urban population. Chest. 2000;117(5):1324 1329.

13. Levine M, Iliescu ME, Margellos-Anast H, Estarziau M, Ansell DA. The effects of cocaine and heroin use on intubation rates and hospital utilization in patients with acute asthma exacerbations. Chest. 2005;128(4):1951-1957.

\section{Author Affiliations}

Christine Y. Zhou, BS; *Melissa Ricker, PA-C; † and Vikas Pathak, MDf, $\S$

*Department of Medicine, Campbell University School of Osteopathic Medicine, Lillington, North Carolina, USA tDepartment of Pulmonary and Critical Care, Atrium Health, Charlotte, North Carolina, USA $¥$ Department of Pulmonary and Critical Care, WakeMed Hospitals and Health System, Raleigh, North Carolina, USA §Department of Medicine, Campbell University School of Osteopathic Medicine, Lillington, North Carolina, USA 